\title{
Transitions of Care in Continuity Clinic-Lessons Learned and Next Steps
}

\author{
Kris $G$. Thomas, MD, FACP \\ Division of Primary Care Internal Medicine, Mayo Clinic, Rochester, MN, USA.
}

J Gen Intern Med 30(11):1574-6

DOI: $10.1007 / \mathrm{s} 11606-015-3413-4$

(c) Society of General Internal Medicine 2015

$\mathrm{T}$ ransitions of care are associated with increased potential for medical errors, adverse patient outcomes, and reduced patient safety. Acknowledged as one of the largest annual synchronous care transitions in this country, outpatient care for an estimated 1 million patients is transitioned from a graduating third year resident to an incoming intern at the start of every academic year. ${ }^{1}$ Patients transitioning care from a graduating resident describe challenges related to establishing a new patient-physician relationship, navigating clinic logistics and care transition processes, and dealing with lapses in care with implications for patient safety. ${ }^{2}$ Following these care transitions, high rates of missed appointments, discontinuity with the newly assigned physician, and loss to follow-up have been observed. ${ }^{3,4}$

From the resident perspective, trainees with less clinical experience and limited interaction with local systems of care are expected to assume care for a panel of patients whom they have never met and who often carry a complex burden of medical and social ailments. Not surprisingly, nearly half of residents do not feel personal responsibility for their assigned patients until they are seen by them in clinic. ${ }^{4}$ This lack of ownership and accountability for patients almost certainly inhibits timely and efficient responses to patient messages, prescription requests, and other care issues that may be needed in the absence of a clinical appointment. Further, junior residents often have limited clinical calendars as compared to their graduated senior resident counterparts, which likely impedes appointment access, prolongs the establishment of patientphysician relationships, and magnifies the consequences of perceived lack of responsibility.

In this issue of JGIM, Solomon et al. report a retrospective cohort study assessing the impact of resident graduation on acute care utilization by residents' primary care patients. ${ }^{5}$ Rates of clinic visits, emergency department (ED) visits, and hospitalizations were assessed before and after the end-of-year transition date and were compared between graduating residents' patients and non-graduating residents' patients. As

Published online May 30, 2015 stated by the authors, this is the first study to use a control group of non-transitioning patients to assess outcomes associated with this resident-patient transition. Using this design, the authors found that transitioning patients had no increase or decrease in the rate of clinic visits, ED visits, or hospitalizations relative to non-transitioning patients.

While the lack of association between acute care utilization and resident graduation reported in this study is reassuring, the authors appropriately recognize the need for continued efforts to standardize outpatient panel transitions, citing other potential impacts on patients, residents, and clinic staff. Compatible with this approach, several initial efforts to improve end-ofyear panel transitions have been reported. These include reducing the variability in the initial outpatient caseload of incoming residents $;{ }^{6}$ integrating patient-centered transition information; ${ }^{7}$ standardizing templates for electronic, written and verbal sign-out; ${ }^{8,9}$ and incorporating a multifaceted approach for transition of high-risk patients. ${ }^{10,11}$ Given the high level of medical and social complexity typical of patients seen in resident clinic, targeted interventions aimed at improving care transitions for high-risk patients may be especially relevant.

Of particular interest in the study by Solomon et al., high rates of acute care utilization were observed throughout the study including both the pre- and post-graduation time intervals and at levels that exceeded national averages. Not surprisingly, higher pre-transition visit rates and higher comorbidity scores were independent predictors of posttransition clinic visits, ED visits, and hospitalizations. Precisely what factors contribute to the overall high rates of acute care utilization observed in this study are unknown. The authors postulate that medical complexity, low socioeconomic status, and limited awareness of clinic resources may all contribute, and they appropriately suggest the need for interventions to decrease acute care utilization throughout the year. Consideration could be given to patientcentered interventions aimed at identifying and reducing barriers to obtaining care in the resident clinic setting; systems interventions targeting proactive scheduling and provision of routine preventive care and chronic disease management and facilitating access for acute medical problems; and education interventions designed to improve residents' skills in outpatient management. Efforts to measure and improve continuity of care could also be 
considered. Rates of patient-physician continuity were not assessed in this study, but continuity may play an important role in acute care utilization, given its association with decreased hospitalizations and emergency department visits. $^{12,13}$

Recognizing the importance of care transitions for patient safety and medical education, the Accreditation Council for Graduate Medicine Education (ACGME) mandates that internal medicine residency programs ensure and monitor effective handoff processes. Transitions in care are a focus area for Clinical Learning Environment Review (CLER). ${ }^{14}$ Based on the research efforts of Solomon et al. and others, patient handoffs and transitions of care are increasingly being recognized as relevant to outpatient in addition to inpatient settings. Additional research is needed to better understand the nature and magnitude of problems associated with outpatient care transitions in order to effectively engage in targeted quality improvement efforts.

First, additional research is needed to determine whether these findings are generalizable to other resident clinic settings and to assess other relevant patient and educational outcomes associated with outpatient panel transitions. Ideally, these would include multi-institutional studies, using control groups for comparison, and assessing clinical outcomes, educational outcomes, and patient and resident perceptions. Patient outcomes are particularly important and could include both process metrics (such as chronic disease surveillance testing, preventive screening, timely medication refill, and follow-up of test results), outcome metrics (such as achievement of target blood pressure or hemoglobin A1c levels), and patient satisfaction metrics.

Second, once the problems associated with outpatient panel transitions are adequately defined and quantified, focused interventions for improvement should be developed and assessed. As discussed, early efforts to improve end-of-year outpatient panel transitions have been described. Continued innovation and rigorous assessment of relevant patient and educational outcomes achieved with these innovations will be important. As exemplified in one prior study, the sustainability of improvement in outpatient panel transition outcomes should also be assessed. ${ }^{11}$

Finally, achieving patient-physician continuity in resident clinic is challenging. Considering the inverse relationship between continuity and transitions of care, and separate from the annual outpatient panel transition of care that was the focus of this study, routine outpatient-to-outpatient care transitions represent an under-recognized, but potentially important patient care transition. The concepts of outpatient-to-outpatient care transitions and outpatient team-based care are especially relevant in the context of recent resident clinic redesign efforts incorporating ' $\mathrm{X}+\mathrm{Y}$ ' models ${ }^{15,16}$ of training in which residents frequently transition to and from inpatient and outpatient settings, often sharing responsibility for cross-coverage of outpatients with other members of their resident clinic team. Analogous to inpatient duty hours, which result in increased handoffs on inpatient services, outpatient ' $\mathrm{X}+\mathrm{Y}$ ' models may increase the need for transitions of care between resident providers in the outpatient clinic setting. Outpatient-to-outpatient transitions of care between providers in the resident continuity clinic setting are not well understood. While not a focus of this study, routine outpatient-to-outpatient care transitions represent another area for further research. Continued efforts to better understand, assess, and improve continuity of care in resident clinic settings are also needed.

In summary, the paper by Solomon et al. makes a valuable contribution to the literature by describing a retrospective cohort study assessing important patient care outcomes in relation to outpatient panel transitions at the time of resident graduation. This is an important step in furthering our understanding of the potential impact of outpatient panel transitions and should inspire more rigorous assessment of this important care transition process. Research on outpatient care transitions is in its relative infancy. Progress is being made, and there remains much yet to be discovered, with the ultimate goal of enhancing and improving patient care and resident education.

Disclaimers: The views expressed in this article are my own and do not reflect the views of Mayo Clinic or any other institution.

Conflict of Interest: I have no conflicts of interest to disclose.

Corresponding Author: Kris G. Thomas, MD, FACP; Division of Primary Care Internal Medicine, Mayo Clinic, 200-1st Street SW, Rochester, MN 55905, USA (e-mail: thomas.kris@mayo.edu).

\section{REFERENCES}

1. Young JQ, Wachter RM. Academic year-end transfers of outpatients from outgoing to incoming residents: an unaddressed patient safety issue. JAMA. 2009;302:1327-9.

2. Pincavage AT, Lee WW, Beiting KJ, Arora VM. What do patients think about year-end resident continuity clinic handoffs? A qualitative study. J Gen Intern Med. 2013;28:999-1007.

3. Caines LC, Brockmeyer DM, Tess AV, Kim H, Kriegel G, Bates CK. The revolving door of resident continuity practice: identifying gaps in transitions of care. J Gen Intern Med. 2011;26:995-8.

4. Pincavage AT, Ratner S, Prochaska ML, et al. Outcomes for residentidentified high-risk patients and resident perspectives of year-end continuity clinic handoffs. J Gen Intern Med. 2012;27:1438-44.

5. Solomon SRGH, Reyes Nieva H, Linder JA. Acute Care Utilization after Graduation of Their Resident Primary Care Physicians. J Gen Intern Med. 2015. doi:10.1007/s11606-015-3305-7.

6. Young JQ, Niehaus B, Lieu SC, O'Sullivan PS. Improving resident education and patient safety: a method to balance initial caseloads at academic year-end transfer. Acad Med. 2010;85:1418-24.

7. Pincavage AT, Lee WW, Venable LR, et al. "Ms. B changes doctors": using a comic and patient transition packet to engineer patient-oriented clinic handoffs (EPOCH). J Gen Intern Med. 2015;30:257-60.

8. Donnelly MJ, Clauser JM, Tractenberg RE. A multicenter intervention to improve ambulatory care handoffs at the end of residency. J Grad Med Educ. 2014;6:112-6.

9. Garment AR, Lee WW, Harris C, Phillips-Caesar E. Development of a structured year-end sign-out program in an outpatient continuity practice. J Gen Intern Med. 2013;28:114-20.

10. Pincavage AT, Dahlstrom M, Prochaska M, et al. Results of an enhanced clinic handoff and resident education on resident patient ownership and patient safety. Acad Med. 2013;88:795-801. 
11. Pincavage AT, Prochaska M, Dahlstrom M, et al. Patient safety outcomes after two years of an enhanced internal medicine residency clinic handoff. Am J Med. 2014;127:96-9.

12. Nyweide DJ, Anthony DL, Bynum JP, et al. Continuity of care and the risk of preventable hospitalization in older adults. JAMA Intern Med. 2013;173:1879-85.

13. Chaiyachati KH, Gordon $\mathbf{K}$, Long $\mathbf{T}$, et al. Continuity in a VA patientcentered medical home reduces emergency department visits. PLoS One. 2014;9:e96356.
14. Weiss KB, Wagner R, Nasca TJ. Development, testing, and implementation of the ACGME Clinical Learning Environment Review (CLER) Program. J Grad Med Educ. 2012;4:396-8.

15. Wieland ML, Halvorsen AJ, Chaudhry R, Reed DA, McDonald FS, Thomas KG. An evaluation of internal medicine residency continuity clinic redesign to a 50/50 outpatient-inpatient model. J Gen Intern Med. 2013;28:1014-9.

16. Mariotti JL, Shalaby M, Fitzgibbons JP. The 4ratiol schedule: a novel template for internal medicine residencies. J Grad Med Educ. 2010;2:541- 